\title{
ARTí́CULO
}

\section{Efecto de la radiación UV sobre la fotosíntesis de Zostera chilensis, proveniente de dos localidades del norte de Chile}

\author{
Effects of UV radiation on photosynthesis of Zostera chilensis, \\ from two location of northern Chile
}

\section{Carolina Sandoval ${ }^{1}$ y Mario Edding ${ }^{1,2}$}

\begin{abstract}
'Departamento de Biología Marina, Facultad de Ciencias del Mar, Universidad Católica del Norte, Larrondo 1281, Coquimbo, Chile.csm018@ucn.cl

${ }^{2}$ CIDTA, Centro de Investigación y Desarrollo Tecnológico en Algas, Facultad de Ciencias del Mar, Universidad Católica del Norte, Larrondo 1281, Coquimbo, Chile
\end{abstract}

\begin{abstract}
Solar radiation affects the biological and chemical processes on the earth surface. The effect of ultraviolet radiation has been analyzed in marine ecosystems by decreasing the ozone layer, including effects on marine angiosperms. The seagrass beds are ecologically important and productive ecosystems. In Chile the seagrass studies are limited. The objective was to evaluate the effect of UV radiation on the photosynthetic efficiency of Zostera chilensis. Samples in austral summer and winter in Puerto Aldea $\left(30^{\circ} \mathrm{S}\right)$ and Salado Bay $\left(27^{\circ} \mathrm{S}\right)$ were collected. The collected material was put on outdoor tanksand using light filters the following conditions were created: PAR + UV; PAR and control (without filter), initial measurements were made and 7 days after final measurements. The daily cycle of photosynthesis in Z. chilensis was also measured. For each treatment and the daily cycle of photosynthesis, the photosynthetic efficiency was evaluated through the Fv/Fm parameter using a portable fluorometer. The results showed no differences in the photosynthetic efficiency of plants excluding UV radiation compared to UV exposed, and with respect to the Fv/Fm initial. In the daily cycle higher Fv/ $\mathrm{Fm}$ were observed in the morning and evening, and a decrease of $\mathrm{Fv} / \mathrm{Fm}$ at noon. Z. chilensis photosynthesis is not sensitive to UV radiation and under high solar radiation, photoinhibition mechanism reducing photosynthetic capacity is developed.
\end{abstract}

Key words: Photosynthesis, UV radiation, Zostera, seagrasses

\begin{abstract}
Resumen.- La radiación solar afecta los procesos biológicos y químicos en la superficie de la tierra. El efecto de la radiación ultravioleta ha sido analizado en los ecosistemas marinos por la disminución de la capa de ozono. Las praderas de pastos marinos son ecosistemas productivos e importantes ecológicamente. Sin embargo, en Chile los estudios en estas especies son limitados. El objetivo del trabajo fue evaluar el efecto de la radiación UV en la eficiencia fotosintética de Zostera chilensis. Para esto, se colectaron muestras en verano e invierno de las localidades de Puerto Aldea ( $\left.30^{\circ} \mathrm{S}\right)$ y Bahía Salado $\left(27^{\circ} \mathrm{S}\right)$. El material colectado fue puesto en estanque al aire libre y con filtros se crearon las condiciones: PAR+UV; PAR y control (sin filtro), se hicieron mediciones iniciales y posteriores a 7 días. Se midió además el ciclo diario de fotosíntesis para Z. chilensis. Para cada tratamiento y para el ciclo diario de fotosíntesis se evaluó la eficiencia fotosintética a través del parámetro Fv/Fm utilizando un fluorómetro portátil. Los resultados muestran que no hay diferencias en la eficiencia fotosintética de aquellas plantas excluidas de radiación UV en comparación a las expuestas al UV. Los valores Fv/Fm iniciales son mayores en invierno comparado con el verano. En el ciclo diario se observan mayores valores $\mathrm{Fv} / \mathrm{Fm}$ en la mañana y al atardecer, y una disminución del Fv/Fm al medio día. La fotosíntesis en Z. chilensis no es sensible a radiación UV y en las horas con mayor radiación solar se desarrolla un mecanismo de fotoinhibición que reduce su eficiencia fotosintética.
\end{abstract}

Palabras clave: Fotosíntesis, radiación UV, Zostera, pastos marinos

\section{INTRODUCCIÓN}

Las pastos marinos pertenecen a un grupo de angiospermas acuáticas adaptadas completamente al ambiente marino. Compiten por luz y sustrato exitosamente con otros organismos marinos fotosintetizadores y poseen un rol de estructuradores de comunidades ya que cubren áreas costeras formando praderas que albergan una gran cantidad de especies, incluidas especies de importancia comercial (Hemminga \& Duarte 2000). Además, los pastos marinos son una importante fuente de producción primaria alrededor del mundo (Zimmerman 
2006). En el norte de Chile se han registrado 2 praderas de pastos (González \& Edding 1990a, b), pero son escasos los estudios que se han realizado en ellas.

La radiación solar afecta los procesos biológicos y químicos que ocurren en la superficie de la tierra (Madronich et al. 1998). Es por esto, desde que se reportó la disminución de la capa de ozono a principios de los años ' 80 , los efectos de la radiación fotosintéticamente activa (PAR) y la radiación (UV) han sido ampliamente analizados en los organismos autótrofos de los ecosistemas marinos (Bischof \& Steinhoff 2012), incluyendo efectos tanto sobre los pastos marinos (Short \& Neckles 1999), como en sobrevivencia y asentamiento de algunos invertebrados marinos (Biermann et al. 1990, Gosselin \& Jones 2010)

Diversos autores han estudiado el efecto de la radiación UV en la fotosíntesis de los pastos marinos encontrando disminución significativa de la eficiencia fotosintética, baja recuperación de fotoinhibición (Dawson \& Dennison 1996, Larkum et al. 2001, Figueroa et al. 2002), disminución de antioxidantes (Hupel et al. 2011) e incluso disminución en la biomasa de algunas praderas de pastos marinos (Mazzuca et al. 2009). Sin embargo, otros estudios demuestran que especies de pasto marino están completamente adaptadas a la radiación UV (Dawson \& Dennison 1996), e incluso en algunas especies poco profundas, la radiación UV-B induce los procesos de recuperación fotosintética y los mecanismo de fotoprotección (Figueroa et al. 2002).

Un método efectivo para medir la fotosíntesis es mediante la fluorescencia de la clorofila $a$, siendo utilizado exitosamente en angiospermas marinas (Horn 2006). El análisis de la fluorescencia de la clorofila $a$ refleja los procesos primarios de la fotosíntesis, tales como la absorción, disipación y reacción fotoquímica en el fotosistema II. Un importante parámetro que refleja el estado fisiológico del aparato fotosintético es la máxima fluorescencia o rendimiento cuántico máximo $(\mathrm{Fv} / \mathrm{Fm})$, que es la razón de (Fm-Fo)/Fm, donde Fm es la eficiencia máxima de la fluorescencia y Fo la eficiencia basal. Una reducción de la eficiencia fotosintética tiene como resultado un decrecimiento en la razón de la variable de máxima fluorescencia (Krause \& Weis 1991). Por lo tanto, una disminución del valor de Fv/Fm representa una disminución en la eficiencia del fotosistema II (PS II).
Estudios del efecto de la radiación ultravioleta en la fotosíntesis del pasto marino Zostera chilensis no se han llevado a cabo. Debido a que el UV afecta la fisiología de los pastos marinos, la máxima fluorescencia debiese aumentar al eliminarse la radiación UV de la radiación incidente sobre el Z. chilensis. Por lo tanto, Z. chilensis aumentará su eficiencia fotosintética en ausencia de radiación UV incidente, independiente de la estacionalidad. El objetivo del estudio fue evaluar el efecto de la radiación ultravioleta en la fotosíntesis de $Z$. chilensis, en 2 estaciones diferentes y proveniente de 2 localidades del norte de Chile y determinar el mecanismo de fotoinhibición diaria, en las estaciones de invierno y verano.

\section{MATERIALES Y MÉTODOS}

\section{Colecta de material}

La zona de colecta comprendió 2 localidades: Puerto Aldea $\left(30^{\circ} 17,524^{\prime} \mathrm{S} ; 7^{\circ} 36,482^{\prime} \mathrm{W}\right)$ ubicada a $58 \mathrm{~km}$ al sur de Coquimbo, IV región de Chile, y Bahía Salado (27²40,039'S; $70^{\circ} 57,344^{\prime} \mathrm{W}$ ) ubicada a $90 \mathrm{~km}$ al sur poniente de Copiapó, III región de Chile, en donde en ambas localidades presentan registros de la especie (González \& Edding 1990a, b). Las muestras de Zostera chilensis fueron colectadas en las estaciones de invierno y verano entre el 2012-2013. En cada localidad y estación se removieron 4 parches de $1,75 \mathrm{~cm}^{3}$ de forma aleatoria en el intermareal de cada pradera, colocados en mallas de nylon y transportados en contenedores térmicos al Laboratorio de Botánica Marina de la Universidad Católica del Norte (Coquimbo, Chile), las cuales fueron mantenidos en estanques de $1000 \mathrm{~L}$ con flujo de aire y agua de mar circulante, durante 48-72 h bajo malla raché en un período de aclimatación a las condiciones del estanque.

\section{EXPERIMENTO DE RADIACIÓN UV}

Dentro de un estanque de $1000 \mathrm{~L}$ (1,8 $\mathrm{m}$ de largo, 0,8 $\mathrm{m}$ de ancho y $0,7 \mathrm{~m}$ de alto), las plantas se dispusieron dentro de 3 contenedores con diferentes condiciones de radiación. Se usaron filtros para crear 2 condiciones: i) PAR+UV-A+UV-B (Ultraphan, $395 \mathrm{~nm}, 0,3 \mathrm{~nm}$, Digefra, Germany) y ii) PAR (Ultraphan, $395 \mathrm{~nm}, 0,12 \mathrm{~nm}$, Munich, Germany), además un control (sin filtro). La radiación PAR, UV-A y UV-B fue registrada diariamente en intervalos de 15 min con un LI-1400 data logger (LI-COR Bioscience Lincoln, NE, USA). 


\section{MEDIDAS DE FLUORESCENCIA}

Para determinar el daño en el fotosistema II (PS II), se utilizó el rendimiento cuántico máximo (Fv/Fm) como parámetro de fluorescencia de la clorofila $a$, el que se midió usando un fluorómetro portátil (PAM 2500, WALZ, Alemania). Para el experimento de UV, 30 mediciones de fluorescencia independientes fueron tomadas inicialmente y luego de 7 días 30 mediciones por tratamiento, previa aclimatación de las hojas a la oscuridad por $20 \mathrm{~min}$.

\section{Ciclo diario de Fotosíntesis}

Ejemplares de Z. chilensis (parches de $1,75 \mathrm{~cm}^{3}$ ) independientes del experimento UV, fueron incubadas en estanque al aire libre de $1000 \mathrm{~L} \mathrm{(1,8} \mathrm{m}$ de largo, 0,8 $\mathrm{m}$ de ancho y $0,7 \mathrm{~m}$ de alto), con flujo de aire y agua de mar circulante. Cada 2 h, desde las 8:00-20:00 h, 30 hojas fueron llevadas al laboratorio donde se midió el Fv/Fm, previa aclimatación a la oscuridad por $15 \mathrm{~min}$. Las mediciones se realizaron en las estaciones de invierno (02/08/2013) y verano (30/01/2014)

\section{Análisis eSTadístico}

Para evaluar la existencia de diferencias estadísticas significativas en el efecto de la radiación UV en la fotosíntesis de Z. chilensis, se aplicó un ANDEVA de dos vías para los factores estación y tipo de radiación en cada localidad por separado y la prueba a posteriori de comparaciones múltiples de Tukey. Para el ciclo diario se aplicó un ANDEVA de dos vías para los factores estación y hora del día. Todas las pruebas estadísticas fueron hechas en el software STATISTICA 7 (StatSoft).

\section{Resultados}

\section{Efecto de la radiación UV en la fotosíntesis de $\boldsymbol{Z}$. CHILENSIS}

La Fig. 1 muestra los valores promedios de Fv/Fm de las diferentes estaciones en las localidades de Puerto Aldea y Bahía Salado. Los valores iniciales de Fv/Fm fueron menores en verano comparado con invierno en las 2 localidades, donde estas diferencias fueron mayores en las plantas provenientes de Bahía Salado.

En las plantas provenientes de Puerto Aldea y Bahía Salado, los valores de Fv/Fm de los distintos tratamientos, comparando las 2 estaciones evaluadas, difieren significativamente entre ellos (Fig. 1). Para las plantas provenientes de la pradera de Puerto Aldea en verano, las eficiencias fotosintéticas en los tratamientos PAR+UV y PAR no fueron diferentes entre ellos $\left(\mathrm{F}_{3,24}=2,66, P\right.$ > $0,05)$, pero si fueron menores significativamente con aquellos valores de Fv/Fm inicial y control (Fig. 1). Sin embargo, en invierno los valores de Fv/Fm de los distintos tratamientos fueron similares, sin presentar diferencias significativas entre ellos (Fig. 1) $\left(\mathrm{F}_{3,24}=2,71, P>0,05\right)$. En la pradera de Bahía Salado, en verano los tratamientos difieren entre ellos, observándose un menor valor de Fv/ Fm en el tratamiento PAR+UV con respecto al tratamiento PAR $\left(\mathrm{F}_{3,24}=14,03, P<0,05\right)$. En invierno, los tratamientos control y PAR+UV presentaron un valor de Fv/Fm menor que el inicial y aquel registrado en el tratamiento PAR, siendo el tratamiento $\mathrm{PAR}+\mathrm{UV}$ el que presentó el menor valor de Fv/Fm $\left(\mathrm{F}_{3,24}=14,03, P<0,05\right)$ (Fig. 1, Tabla 1).

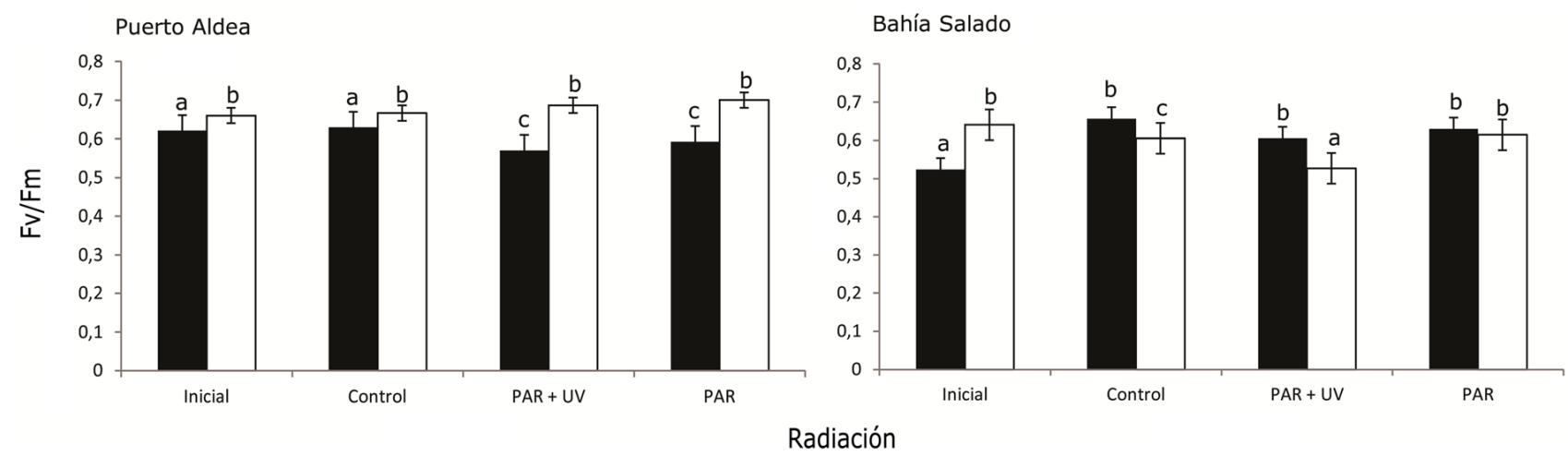

Figura 1. Eficiencia fotosintética Fv/ Fm (media $\pm \mathrm{DE}$ ) en verano (barras negras) e invierno (barras blancas). Las letras minúsculas representan valores significativamente iguales (Tukey, $\mathbf{P}<\mathbf{0 , 0 5}$ ) / Photosynthetic efficiency Fv/Fm (mean \pm SD) summer (black bars) and winter (white bars). Lowercase letters represent significantly equal values (Tukey, $\mathrm{P}<0.05$ ) 
Tabla 1. Valores (media $\pm \mathrm{DE}$ ) de rendimiento cuántico máximo $(\mathrm{Fv} / \mathrm{Fm})$ determinados en las 4 condiciones estudiadas para plantas de $Z$. chilensis provenientes de $\mathbf{2}$ localidades y $\mathbf{2}$ estaciones del año. Valores seguidos por letras minúsculas iguales no hubo diferencia significativa / Values (mean $\pm \mathrm{SD}$ ) maximum quantum yield ( $\mathrm{Fv} / \mathrm{Fm})$ of 4 treatments for $\mathrm{Z}$. chilensis plants from 2 locations and 2 seasons. The same lowercase letters correspond to values without significant differences

\begin{tabular}{lcccc}
\hline & Iniciales & PAR + UV & PAR & Control \\
\hline Plantas Puerto Aldea & & & & \\
$\quad$ Invierno & $0,6601 \pm 0,02^{\mathrm{b}}$ & $0,6865 \pm 0,02^{\mathrm{b}}$ & $0,7003 \pm 0,03^{\mathrm{b}}$ & $0,6635 \pm 0,02^{\mathrm{b}}$ \\
$\quad$ Verano & $0,6209 \pm 0,04^{\mathrm{a}}$ & $0,5698 \pm 0,04^{\mathrm{c}}$ & $0,5929 \pm 0,03^{\mathrm{c}}$ & $0,5541 \pm 0,07^{\mathrm{a}}$ \\
& & & & \\
Plantas Bahía Salado & & & & \\
$\quad$ Invierno & $0,6403 \pm 0,05^{\mathrm{b}}$ & $0,5270 \pm 0,04^{\mathrm{a}}$ & $0,6145 \pm 0,03^{\mathrm{b}}$ & $0,6055 \pm 0,04^{\mathrm{c}}$ \\
$\quad$ Verano & $0,5238 \pm 0,05^{\mathrm{a}}$ & $0,6051 \pm 0,03^{\mathrm{b}}$ & $0,6293 \pm 0,03^{\mathrm{b}}$ & $0,6564 \pm 0,02^{\mathrm{b}}$ \\
\hline
\end{tabular}

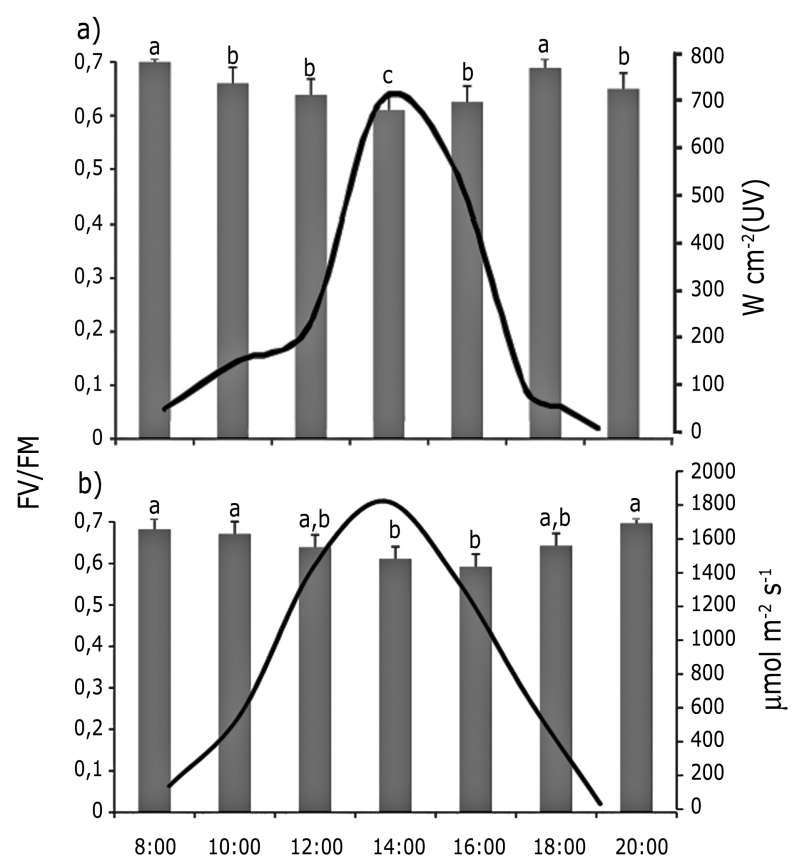

Figura 2. Ciclo diario de fotosíntesis en plantas de Puerto Aldea. a) invierno $\mathrm{y}$ b) verano. Las barras representan el valor de medio $\pm D E$ $(n=30)$ de $\mathrm{Fv} / \mathrm{Fm}$ y la línea continua la radiación PAR / Daily cycle of photosynthesis from Puerto Aldea plants. A) winter and B) summer. The bars represent the values mean $\pm S D(n=30)$ of $F v /$ $\mathrm{Fm}$ and the continues line the PAR radiation

\section{Ciclo diario de fotosíntesis}

El ciclo diario de fotosíntesis de las plantas de Puerto Aldea presentó una disminución en la eficiencia fotosintética en las horas con mayor radiación y un aumento en las horas con menor radiación (Fig. 2) (Tabla 1). En invierno, los mayores valores de $\mathrm{Fv} / \mathrm{Fm}$ fueron de $0,70 \pm 0,02$ y $0,68 \pm 0,03$ a las $8: 00$ y $18: 00 \mathrm{~h}$ respectivamente, mientras que el menor valor de Fv/Fm fue de 0,60 \pm 0,03 a las 14:00 h (Fig. 2a, Tabla 1). En verano, los mayores valores de $\mathrm{Fv} / \mathrm{Fm}$ fueron de $0,68 \pm 0,05$ y $0,70 \pm 0,03$ a las 8:00 y 20:00 h respectivamente, mientras que el menor valor de Fv/Fm fue 0,59 \pm 0,07 a las 16:00 h (Fig. 2b) (Tabla 1). Existen diferencias significativas en los valores de Fv/ Fm de un mismo día $\left(\mathrm{F}_{5,56}=3,872, P<0,05\right)$, así como se registran diferencias significativas en los valores de Fv/ Fm entre las estaciones de invierno y verano $\left(\mathrm{F}_{5,56}=0,695\right.$, $P>0,05)$.

\section{Discusión}

Los resultados evidencian que Zostera chilensis es una especie poco sensible a la radiación UV en las condiciones del estudio. En general, no se detectó un efecto de la radiación UV (presencia o ausencia) sobre la eficiencia fotosintética. Sin embargo, la estacionalidad fue un factor que influyó en la fotosíntesis de plantas de la pradera de Puerto Aldea, observándose una eficiencia fotosintética máxima en invierno en comparación al verano. Estudios del pasto marino Cymodoceae surrelata muestran que esta especie también presenta una alta tolerancia fisiológica al incremento de UV (Dawson \& Dennison 1996). En Cymodea nodosa se observó que la eficiencia fotosintética y fotosíntesis máxima fueron afectadas por la estacionalidad, existiendo una mayor fotosíntesis durante verano (Olivé et al. 2013) La transferencia de plantas submareales a la zona intermareal, han demostrado que plantas de pastos marinos se adaptan eficientemente al aumento de radiación (Häder et al. 2007). Se ha determinado que para protegerse del daño celular causado por la radiación UV, los organismos aumentan la síntesis de proteínas y componentes celulares que generan 
mecanismos de fotoprotección, teniendo como consecuencia una reducción en la actividad fotosintética conocida como fotoinhibición (Hanelt \& Figueroa 2012). Las hojas de Z. chilensis están altamente epifitadas por Rhodophyta filamentosas, las cuales absorben luz PAR y nutrientes limitando las plantas epifitadas, pero también se ha considerado que el crecimiento de epífitas sobre las hojas es un factor determinante para reducir la radiación absorbiendo el UV (Häder et al. 2007). La combinación de moléculas fotoprotectoras y el beneficio ejercido por las epifitas podrías estar contribuyendo a que $Z$. chilensis tenga poca sensibilidad a la radiación UV.

El ciclo diario de fotosíntesis de Zostera chilensis sigue el mismo patrón detectado en algas rojas, pardas y otras angiospermas, en donde se registra un decrecimiento de la fotosíntesis al mediodía (horas de mayor radiación) y al atardecer (Hanelt 1992, Horn 2006, Hanelt \& Roleda 2009). Ésta dinámica de fotoinhibición es un mecanismo de fotoprotección, proceso en el que decrece la energía de excitación transferida al centro de reacción de la clorofila (Hanelt \& Figueroa 2012), permitiéndole al organismo adaptarse a condiciones de alta irradiancia. Un importante mecanismo de aclimatación fisicoquímica a la radiación, es la producción de moléculas que absorban o disipen la radiación UV (Bischof \& Steinhoff 2012). Zostera capricorni a pesar de ser sensible al UV muestra una rápida aclimatación incrementando el contenido de pigmentos que absorben el UV al mediodía (Dawson \& Dennison 1996). En Z. chilensis proveniente de la población de Puerto Aldea se encontró más cantidad de carotenoides en plantas que habitan el intermareal alto en comparación a plantas que habitan el submareal (PazSoldán 2008). Se ha observado en muchos autótrofos que la presencia de UV-B es necesaria para activar los mecanismos de fotoinhibición (Hanelt \& Roleda 2009). Por ejemplo, en la angiosperma marina Posidonia oceánica, la ausencia de radiación UV-B resulta en una pérdida de la capacidad de fotoprotección (Figueroa et al. 2002).

Las especies que habitan el intermareal están expuestas a condiciones de alta irradiancia que se incrementa en los periodos de baja marea (Hanelt \& Figueroa 2012). Zostera chilensis es una especie intermareal, en general las angiospermas marinas presentan altos requerimientos de luz. Mientras las algas poseen un requerimiento de radiación en la superficie del mar $\left[\mathrm{E}_{\mathrm{d}}(0)\right]$ entre 0,1 a $1 \%$, los pastos marinos poseen un $\left[\mathrm{E}_{\mathrm{d}}(0)\right]$ de 10 a $37 \%$ (Zimmerman 2006). Sin embargo, algunas Angiospermas marinas habitan el submareal y al ser expuestas a altas radiaciones, su eficiencia fotosintética disminuye (Dawson \& Dennison 1996). Zostera chilensis, posee un requerimiento mínimo de luz de $20 \%$ siendo uno de los más altos entre los pastos marino (Lee et al. 2007), por lo que esta especie estaría completamente adaptada a altas condiciones de luz en los hábitats que ocupa.

A nivel mundial existe una preocupación por la pérdida de praderas de pastos marinos debido a factores antrópicos. Uno de los principales factores de perdida de praderas se producen por el enriquecimiento de materia orgánica producida por la descarga de efluentes urbanos en ambiente marinos, provocando disminución de las disponibilidad de luz (Balestri et al. 2004, Miller et al. 2005). En Chile los estudios de Z. chilensis son escasos y no existe un monitoreo de las praderas que evalúe una pérdida de éstas, aun cuando la especies Z . chilensis se encuentra categorizada como especie en peligro de extinción y en periodo de declinación de la población por la IUCN (Unión Internacional para la conservación de la Naturaleza)

\section{Agradecimientos}

Esta investigación se realizó como parte de la tesis del primer autor, para optar al título de Biólogo Marino, Facultad de Ciencias del Mar. Se agradece el apoyo para la realización del presente trabajo a la Universidad Católica del Norte, al Proyecto FONDEF-CONICYT 'Red de información en biodiversidad para orientar las prioridades en investigación científica en apoyo a las políticas públicas ambientales', y al Centro de Investigación y Desarrollo Tecnológico en Algas (CIDTA).

\section{LITERATURA CITADA}

Balestri E, L Benedetti-Cecchi \& C Lardicci. 2004. Variability in patterns of growth and morphology of Posidonia oceanica exposed to urban and industrial wastes: contrasts with two reference locations. Journal of Experimental Marine Biology and Ecology 308: 1-21.

Biermann H, G Shinner \& R Strathmann. 1990. Influence of solar radiation, microalgal fouling, and current on deposition site and survival of embryos of a dorid nudibranch gastropod. Marine Ecology Progress Series 86: 205-215.

Bischof K \& FS Steinhoff. 2012. Impact of ozone stratospheric depletion and solar UVB radiation on seaweeds. In: Wiencke C \& K Bischof (eds). Seaweed biology: novel insights into ecophysiology, ecology and utilization, pp. 433-448. Springer, Berlin / Heidelberg.

Dawson SP \& WC Dennison.1996. Effects of ultraviolet and photosynthetically active radiation on five seagrass species. Marine Biology 125: 629-638. 
Figueroa F, C Jiménez, B Viñegla, E Pérez-Rodríguez, J Aguilera, A Flores-Moya, M Altamirano, M Lebert \& D Häder. 2002. Effects of solar UV radiation on photosynthesis of the marine angiosperm Posidonia oceanica from southern Spain. Marine Ecology Progress Series 230: 59-70.

González S \& M Edding. 1990a. Extension of range of Heterozostera tasmanica (Martens ex Aschers.) den Hartog in Chile. Aquatic Botany 38: 391-395.

González S \& M Edding. 1990b. Achelia assimilis (Haswell, 1884) in the Heterozostera bed of Puerto Aldea, Coquimbo: First record from the northern Chilean coast (Pycnogonida: Ammotheidae). Proceedings of the Biological Society of Washington 103: 151-156.

Gosselin L \& L Jones. 2010. Effects of solar radiation on barnacle settlement, early post-settlement mortality and community development in the intertidal zone. Marine Ecology Progress Series 407: 149-158.

Hanelt D. 1992. Photoinhibition of photosynthesis in marine macrophytes of the South China Sea. Marine Ecology Progress Series 82: 199-206.

Häder D, H Kumar, R Smith \& R Worrest. 2007. Effects of solar UV radiation on aquatic ecosystems and interactions with climate change. Photochemical and Photobiological Sciences 6: 267-285.

Hanelt D \& FL Figueroa. 2012. Physiological and photomorphogenic effects of light on marine macrophytes. In: Wiencke C \& K Bischof (eds). Seaweed biology: novel insights into ecophysiology, ecology and utilization, pp. 3-24, Springer, New York.

Hanelt D \& MY Roleda. 2009. UVB radiation may ameliorate photoinhibition in specific shallow-water tropical marine macrophytes. Aquatic Botany 91: 6-12.

Hemminga M \& CM Duarte. 2000. Seagrass ecology, 298 pp. Cambridge University Press, Cambridge

Horn LE. 2006. The measurement of seagrass photosynthesis using Pulse amplitude modulated (PAM) fluorometry and its practical applications, specifically in regard to transplantation. Thesis $\mathrm{PhD}$, Biological Sciences and Biotechnology, Murdoch University, Perth, 127 pp. <http:/ /researchrepository.murdoch.edu.au/106/>

Huovinen P, I Gómez, FL Figueroa, N Ulloa, V Morales \& C Lovengreen. 2004. Ultraviolet-absorbing mycosporinelike amino acids in red macroalgae from Chile. Botánica Marina 47: 21-29
Hupel M, C Lecointre, A Meudec, N Poupart \& EA Gall. 2011. Comparison of photoprotective responses to UV radiation in the brown seaweed Pelvetia canaliculata and the marine angiosperm Salicornia ramosissima. Journal of Experimental Marine Biology and Ecology 401: 36-47.

Krause G \& E Weis. 1991. Chlorophyll fluorescence and photosynthesis: The Basics. Annual Review of Plant Physiology and Plant Molecular Biology 42: 313-349.

Larkum W, M Karge, F Reifarth, HJ Eckert \& G Renger. 2001. Effect of monochromatic UV-B radiation on electron transfer reactions of Photosystem II. Photosynthesis Research 68: 49-60.

Lee K-S, SR Park \& YK Kim. 2007. Effects of irradiance, temperature, and nutrients on growth dynamics of seagrasses: A review. Journal of Experimental Marine Biology and Ecology 350: 144-175.

Madronich S, RL McKenzie, LO Björn \& MM Caldwell. 1998. Changes in biologically active ultraviolet radiation reaching the Earth's surface. Journal of Photochemistry and Photobiology 46: 5-19.

Mazzuca S, A Spadafora, D Filadoro, C Vannini, M Marsoni, R Cozza, M Bracale, T Pangaro \& A Maria. 2009. Seagrass light acclimation/: 2 -DE protein analysis in Posidonia leaves grown in chronic low light conditions. Journal of Experimental Marine Biology and Ecology 374: 113-122.

Miller C, S Campbell \& S Scudds. 2005. Spatial variation of Zostera tasmanica morphology and structure across an environmental gradient. Marine Ecology Progress Series 304: 45-53.

Olivé I, J Vergara \& J Pérez-Lloréns. 2013. Photosynthetic and morphological photoacclimation of the seagrass Cymodocea nodosa to season, depth and leaf position. Marine Biology 160: 285-297.

Paz-Soldán C. 2008. Comparación estacional y batimétrica de parámetros fisiológicos del pasto marino Heterozostera chilensis J Kuo (2005), en Puerto Aldea, Región de Coquimbo, Chile. Tesis de Biología Marina, Facultad de Ciencias del Mar, Universidad Católica del Norte, Coquimbo, $72 \mathrm{pp}$.

Short FT \& H Neckles. 1999. The effects of global climate change on seagrasses. Aquatic Botany 63: 169-196.

Zimmerman RC. 2006. Light and photosynthesis in seagrass meadows. In: Larkum A, R Orth \& CM Duarte (eds). Seagrasses: biology, ecology and conservation, pp. 303321. Springer, Dordrecht.

Recibido el 23 de mayo de 2014 y aceptado el 3 de marzo de 2015

Editor asociado: Loretto Contreras Porcia 